\title{
Stirred Suspension Culture Improves Embryoid Body Formation and Cardiogenic Differentiation of Genetically Modified Embryonic Stem Cells
}

\author{
Wenjun He,${ }^{a, b}$ Lingling Ye, ${ }^{a}$ Shichong Li, ${ }^{a}$ Hong Liu, ${ }^{a}$ Qiwei Wang, ${ }^{a}$ Xiaobing Fu,,${ }^{b, c}$ \\ Weidong Han, ${ }^{c}$ and Zhaolie Chen*,a \\ ${ }^{a}$ Department of Cell Engineering, Institute of Biotechnology, Academy of Military Medical Sciences; 20 Dongdajie \\ Street, Fengtai District, Beijing 100071, China: ${ }^{b}$ Wound Healing and Cell Biology Laboratory, The First Affiliated \\ Hospital, Chinese PLA General Hospital; 51 Fucheng Road, Haidian District, Beijing 100048, China: and ${ }^{c}$ Institute \\ of Basic Medical Sciences, Chinese PLA General Hospital; 28 Fuxing Road, Haidian District, Beijing 100853, China. \\ Received July 28, 2011; accepted December 6, 2011; published online December 16, 2011
}

Embryonic stem cells (ESCs) can propagate unlimitedly in vitro and differentiate into cardiomyocytes, which have been proposed as unlimited cell sources for cardiac cell therapy. This was limited by difficulties in large-scale generation of pure cardiomyocytes. In this study, we used stirred bioreactors to optimize the differentiation condition for mass production of embryoid bodies (EBs) derived from genetically modified mouse ESCs. Stirred suspension culture could more efficiently produce EBs and have a more uniform EB population without large necrotic centers, compared with the conventional static culture. Importantly, the cardiac-specific gene expressions (GATA binding protein 4, $\alpha$-cardiac myosin heavy chain and myosin light chain-2v) were increased within EBs cultured in stirred bioreactor. Stirred suspension culture significantly increased the proportion of spontaneously contracting EBs, yielded a greater percentage of $\alpha$-sarcomeric actinin-positive cells detected via flow cytometry, and harvested relatively more cardiomyocytes after G418 selection. Stirred suspension culture provided a more ideal culture condition facilitating the growth of EBs and enhancing the cardiogenic differentiation of genetically modified ESCs, which may be valuable in largescale generation of pure cardiomyocytes.

Key words embryonic stem cell; cardiomyocyte; stirred bioreactor; genetic modification; embryoid body

Embryonic stem cells (ESCs) are derived from the inner cell mass of blastocyst stage preimplantation embryos. ${ }^{1)}$ ESCs are capable of self-renewal in vitro and retain the ability to differentiate into various cell types, so they not only provide a valuable model in vitro for developmental biology, but also serve as a potent cell source for tissue engineering and cell therapy. ${ }^{2,3)}$ Because postnatal cardiomyocytes have little or no regenerative capacity, ESCs are a potential cell source to form transplantable bona fide cardiomyocytes in vitro. For most future cell therapy applications, however, large numbers of pure and mature cardiomocytes are will be required.

Although the methods for induction of ESC differentiation into cardiomyocytes have already been reported, ${ }^{4-6)}$ the percentage of cardiomyocytes in ESC-derived differentiated cells was very low. The heterogenous population still contained a large number of non-myocardial cells and some of the undifferentiated ESCs, which would be a serious impediment for clinical application of ES-derived cardiomyocytes. Manual dissection of beating areas and percoll gradient centrifugation have been used to enrich cardiomyocytes, but the degree of purity would probably be insufficient for clinical or research purposes. $^{7,8)}$ Genetic selection may offer an alterative route to enrich cardiomyocytes. To select cardiomyocytes within embryoid bodies (EBs), a vector containing $\alpha$-cardiac myosin heavy chain $(\mathrm{MHC})$ promoter driving a neomycin resistance gene was established. ${ }^{9)}$ After selection using G418, exclusively cardiomyocytes survived and were $>99 \%$ pure based on sarcomeic myosin immunoreactivity. ${ }^{9)}$

Currently, ESC differentiation is usually via induction of EB formation in suspension. The quality of EBs will affect the efficiency of cardiomyocytes differentiation dramatically. Controlling cell number and size of EBs may be an important step in directed cardiac differentiation strategies. Typically, several different methods have been developed for EB formation, such as static liquid suspension culture, methylcellulose culture and hanging drop culture, but they could not meet the quantitative need of exploratory development of tissue engineering and regenerative medicine. When EBs were formed in static suspension culture, it was difficult to control the size and shape of EBs and often resulted in extensive agglomeration of EBs, which caused cell death and had a negative impact on differentiation of EBs. ${ }^{10)}$ Recent studies have confirmed that spinner flask and slow turning lateral vessel bioreactor culture could be used for large-scale production of EBs. ${ }^{1-14)}$ The agitation culture could not only improve circulation of nutrients and cell waste products, but also be manipulated to control cell aggregation. ${ }^{15)}$ Importantly, stirred or rotary EBs appeared to differentiate more efficiently than static suspension cultures on the basis of morphological appearance and gene expression profile patterns. ${ }^{16,17)}$

Based on above problems of cardiogenic differentiation of ESCs, we tried to use stirred bioreactors to optimize the culture condition for genetically modified mouse ESCs in this study. Under the treatment of ascorbic acid, expression of the fusion gene in ESC-derived cardiomyocytes facilitated their selection with G418 after in vitro differentiation. The efficiency of EB formation, homogeneity, yield, and differentiation of EBs were compared between stirred suspension and static suspension culture. In addition, the cardiogenic differentiation of EBs was specifically compared in cardiac-specific gene expression, spontaneous contractile activity, sarcomeric protein expression, and cardiomyocyte yields. 


\section{MATERIALS AND METHODS}

Cell Culture Generation of the genetically modified mouse ESC line MN6 was performed as previously described. ${ }^{18)}$ Briefly, $5 \times 10^{6}$ undifferentiated ESCs (ES-D3) suspended in $800 \mu \mathrm{L}$ phosphate buffered saline (PBS) were transfected via electroporation $(240 \mathrm{~V} / 500 \mu \mathrm{F})$ with $50 \mu \mathrm{g}$ plasmid (pMHC-neo/SV40-hygro) carrying cardiac $\alpha$-MHC promoter in front of the neomycin resistance gene and SV40 promoter in front of the hygromycin resistant gene in a pcDNA3.1(+) vector backbone (Invitrogen). The MN6 cells were maintained in an undifferentiated state by culturing on a feeder layer of mitomycin C-treated mouse embryonic fibroblasts (MEF) on $0.1 \%$ gelatin-coated tissue culture dishes in ESC culture medium containing 85\% high glucose Dulbecco's modified Eagle's medium (DMEM) (Invitrogen), 15\% fetal bovine serum (FBS, Hyclone), $0.1 \mathrm{~mm} \beta$-mercaptoethanol (Sigma), $1 \%$ nonessential amino acids (Invitrogen) and $10^{3} \mathrm{U} / \mathrm{mL}$ leukemia inhibitory factor (LIF, Millipore). The culture medium was changed daily, and were routinely passaged by disassociating with $0.25 \%$ trypsin and $0.04 \%$ ethylenediaminetetraacetic acid (EDTA) every second day and reseeded on freshly prepared MEF feeder layer.

EB Formation To induce EB formation, undifferentiated MN6 ESCs were dispersed into single cells using 0.25\% trypsin and $0.04 \%$ EDTA, and preplated on tissue culture dishes for $60 \mathrm{~min}$ in differentiation medium (ES culture medium without LIF and replacing $15 \%$ for $20 \%$ FBS) to reduce the MEF. To optimize culture conditions for mass production of EBs, two culture systems were compared in the study: (1) $100 \mathrm{~mm}$ Petri dishes with $10 \mathrm{~mL}$ differentiation medium for static culture; (2) $250 \mathrm{~mL}$ stirred bioreactor (Bellco). After resuspension in differentiation medium, MN6 cells were seeded into the stirred bioreactor with initial cell concentrations ranging from $1 \times 10^{3}-1 \times 10^{7}$ cells $/ \mathrm{mL}$ to find the optimal condition. The initial stirring speeds were set at $10-60 \mathrm{rpm}$. With the EB size increasing, the stirring speed should be increased to prevent EBs deposition in the bottom of the stirred bioreactor. During the $5 \mathrm{~d}$ of suspension culture, half of the medium was exchanged with fresh medium every second day.

EB Number and Size Measurement In order to evaluate the efficiency of EB formation, EBs from the two culture systems were transferred into a 96-well plate and counted using an inverted light microscope every day. Ten wells were counted to determine the average EB number of a sample. Spheroids consisting of more than 10 cells were scored as EBs. For size analysis, the average diameter was calculated by measuring the large and small diagonals of eight representative EBs from each field. The results of measurements from two independent experiments are presented.

Detection of Live/dead Cells in EBs To evaluate the quality of EBs formed in the two culture systems, acridine orange/propidium iodide (AO/PI) staining was performed to detect live/dead cells in EBs. Briefly, the stock solution (AO: $670 \mu \mathrm{mol} / \mathrm{L}$, PI: $750 \mu \mathrm{mol} / \mathrm{L})$ was prepared in PBS and kept in the dark at $4^{\circ} \mathrm{C}$. Just before the experiment, $0.01 \mathrm{~mL} A O$ and $1.0 \mathrm{~mL}$ PI were mixed and diluted 10 times with PBS, and then filtered with $0.22 \mu \mathrm{m}$ filter membrane. The 5-d-old EBs sampled from the two culture systems were incubated with $\mathrm{AO} / \mathrm{PI}$ staining solution at room temperature for $10 \mathrm{~min}$, then washed three times with PBS. Samples were transferred to 24-well plate and immediately imaged using a fluorescent microscopy.

Histological Analysis After five days of culture, samples were fixed in $10 \%$ formalin for $30 \mathrm{~min}$, dehydrated in graduated alcohols (70-100\%), and embedded in paraffin. Samples were cut into $4 \mu \mathrm{m}$ thick sections, and hematoxylin and eosin (H\&E) staining was performed according to the regular procedure to observe necrotic centers in the EBs.

Quantitative Reverse Transcription-Polymerase Chain Reaction (RT-PCR) Analysis Total RNA was extracted from undifferentiated MN6 and EBs from the Petri dishes culture and stirred suspension culture on days 1, 3, and 5 after suspension using TRIzol (Invitrogen) according to the specification indicated by the manufacturer. RNA was quantified by UV spectrophotometer. Reverse transcription for cDNA synthesis was performed with $1 \mu \mathrm{g}$ of RNA per sample using the PrimeScript RT-PCR kit (TaKaRa), and quantitative PCR was performed with SYBR green technology on the MyiQ cycler (Bio-Rad). The primers and sequences are listed in Table 1 for GATA binding protein 4 (GATA-4), $\alpha$-MHC, myosin light chain (MLC)-2v, Oct-4, and $\beta$-actin. Relative gene expression level of Oct-4 was quantified relative to the expression level of undifferentiated ESCs using the Pfaffl method of quantification. GATA-4, $\alpha$-MHC and MLC-2v concentrations were calculated using standard curves and normalized to $\beta$-actin expression levels.

Induction of Cardiogenic Differentiation of EBs To induce differentiation of EBs into cardiomyocytes, 5-d-old EBs formed in the two culture systems were respectively inoculated onto $0.1 \%$ gelatin-coated 96 -well or 24 -well plates for the evaluation of the cardiomyocyte differentiation efficiency and immunofluorescent staining, and tissue culture dishes for flow cytometric analysis and cardiomyocytes selection. The differentiation medium was supplemented with $0.1 \mathrm{mg} / \mathrm{mL}$ ascorbic acid (Sigma) to induce EBs differentiation into cardiomyocyte. Nothing was added into the differentiation medium as control to compare the efficiency of cardiogenic differentiation. The cultures were monitored daily under a phase contrast microscope for the presence of contracting cardiomyocytes. The percentage of EBs containing beating cardiomyocytes was determined in three independent experiments after $14 \mathrm{~d}$ of

Table 1. Primer Sequences for Quantitative PCR

\begin{tabular}{cll}
\hline \hline Gene & Forward sequence $\left(5^{\prime}-3^{\prime}\right)$ & Backward sequence $\left(5^{\prime}-3^{\prime}\right)$ \\
\hline GATA-4 & GCACATCACCCATCACACAG & CAGATGAGCCATAGTCAC \\
$\alpha$-MHC & AACATCGCCATCGTGGAACG & CAGGTCTGACTCCATCTTC \\
MLC-2v & CAAAGGGCACTGAAGGC & TCAAGATTGCCGGTGACGTC \\
Oct-4 & CAGCTCAGCCTTAAGAACAT & CAGAAACATGGTCTCCAGAC \\
\hline
\end{tabular}


differentiation.

Immunofluorescence Staining After $14 \mathrm{~d}$ of differentiation, the cells were rinsed briefly with PBS and fixed with 4\% paraformaldehyde at room temperature for $15 \mathrm{~min}$. The fixed cells were washed three times in PBS and further treated with $0.3 \%$ Triton $\mathrm{X}-100$ for $30 \mathrm{~min}$. Unspecific binding sites were blocked by $5 \%$ bovine serum albumin (BSA) for $30 \mathrm{~min}$. The primary antibodies against $\operatorname{cTnT}(1: 100$, Maxin) and $\alpha$-sarcomeric actinin $(1: 100$, Boster $)$ were applied without washing and incubated at $4^{\circ} \mathrm{C}$ overnight. After washing, cells were treated with the secondary antibodies fluorescein isothiocyanate (FITC)- or TRITC-labeled goat anti-mouse immunoglobulin $\mathrm{G}$ (IgG) $(1: 50$, ZSGB-BIO) for $2 \mathrm{~h}$ at room temperature in dark. Nuclei were stained with $0.1 \mu \mathrm{g} / \mathrm{mL}$ of DAPI (Sigma) at room temperature for $15 \mathrm{~min}$. Samples were observed under a confocal microscope (Zeiss).

Flow Cytometric Analysis EBs induced by ascorbic acid for $9 \mathrm{~d}$ (day 14 of differentiation) were washed with PBS, dissociated with $0.25 \%$ trypsin and $0.04 \%$ EDTA, triturated every $10 \mathrm{~min}$ for a total of $30 \mathrm{~min}$, and then passed through a $35 \mu \mathrm{m}$ cell-strainer to remove cell clumps to attain single cell suspensions for flow cytometric analysis. The resultant cell suspensions were centrifuged at $2000 \mathrm{rpm}$ for $5 \mathrm{~min}$ to collect cells, resuspended in $1 \mathrm{~mL}$ PBS, transferred to $1.5 \mathrm{~mL}$ centrifuge tubes, centrifuged again at $4000 \mathrm{rpm}$ for $5 \mathrm{~min}$, and resuspended in $1 \mathrm{~mL}$ of $4 \%$ paraformaldehyde for $10 \mathrm{~min}$ at room temperature. Cells were collected by centrifugation and rinsed three times with PBS, and then were resuspended in $0.3 \%$ Triton $\mathrm{X}-100$ for $30 \mathrm{~min}$. After rinse and centrifugation, $0.5 \%$ BSA was added for block at room temperature. Cells were centrifuged and stained with the primary antibody against $\alpha$-sarcomeric actinin $(1: 100$, Boster $)$ at $37^{\circ} \mathrm{C}$ for $1 \mathrm{~h}$, followed by successive rinse and centrifugation. The cell pellets were resuspended in secondary antibody FITC-labeled goat anti-mouse $\operatorname{IgG}(1: 50, \mathrm{ZSGB}-\mathrm{BIO})$ in the dark for $30 \mathrm{~min}$ at $37^{\circ} \mathrm{C}$, followed by a series of three rinses in PBS and centrifugation. The isotype control antibody used was FITCconjugated mouse IgG1. The final cell pellets were resuspended in $500 \mu \mathrm{L}$ of PBS for flow cytometric analysis.

Cardiomyocytes Selection After $9 \mathrm{~d}$ of induction with ascorbic acid, attached EBs were grown for $8 \mathrm{~d}$ in the presence of G418 (400 $\mu \mathrm{g} / \mathrm{mL}$, Invitrogen) for caridomyocytes selection. To obtain a single cell suspension, EBs were washed with PBS, and then incubated with $0.25 \%$ trypsin and $0.04 \%$ EDTA at $37^{\circ} \mathrm{C}$ for $30 \mathrm{~min}$, followed by cautious mechanical dissociation. After digestion was terminated by FBS, cell suspension was filtered through a $35 \mu \mathrm{m}$ cell-strainer. Count of viable cells was determined via a hemocytometer and trypan blue staining. The cardiomyocytes output was compared between stirred bioreactors and Petri dishes by a coefficient comprising the number of cardiomyocytes generated per input ESC (CM/ ESC).

Statistical Analysis All experiments were performed at least three times. The data were expressed as mean \pm S.D. and analyzed by Student's $t$-test or one-way analysis of variance (ANOVA) with post hoc analysis (Bonferroni). $p<0.05$ was considered statistically significant.

\section{RESULTS}

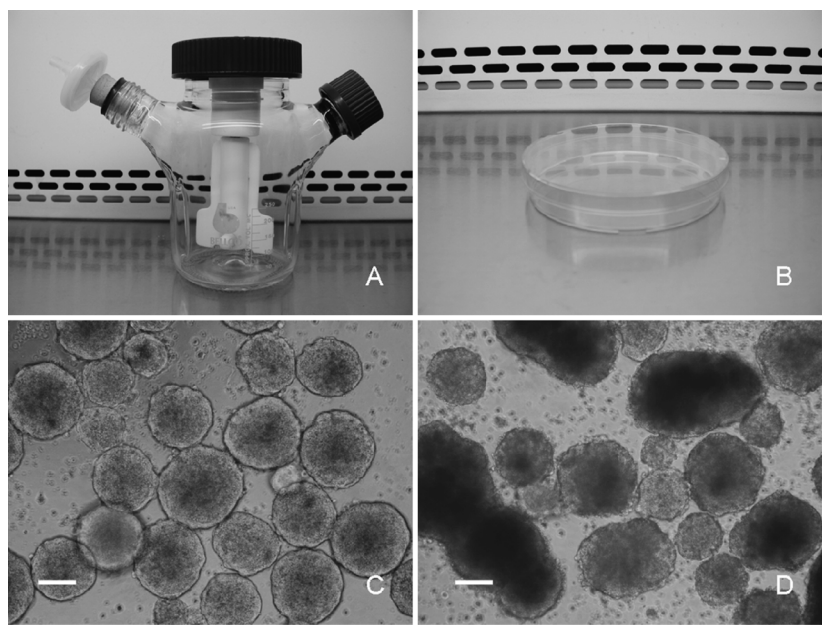

Fig. 1. Formation of EBs in Stirred Bioreactors and Petri Dishes after $5 \mathrm{~d}$ of Suspension Culture

(A) Stirred bioreactor for stirred suspension culture; (B) Petri dish for static suspension culture; (C) EBs formed in stirred bioreactors showed a relatively homogenous population; (D) EBs formed in Petri dishes showed an extensive agglomeration. Scale bars $=200 \mu \mathrm{m}$.

determine the optimal condition for EB formation, the undifferentiated mouse MN6 ESCs were inoculated in $250 \mathrm{~mL}$ stirred bioreactorsfor stirred suspension (Fig. 1A) and $100 \mathrm{~mm}$ conventional Petri dishesfor static suspension (Fig. 1B) at the same initial concentrations ranging from $1 \times 10^{3}-1 \times 10^{7}$ cells/ $\mathrm{mL}$. When seeded with low cell concentrations less than $5 \times 10^{4}$ cells $/ \mathrm{mL}$, no cell aggregates were formed up to $24 \mathrm{~h}$ after seeding; although the stirring speed was minimized to $10 \mathrm{rpm}$, the efficiency of EB formation was low and EBs were not visible clearly. When seeded at a density of more than $1 \times 10^{6}$ cells $/ \mathrm{mL}$, the cells aggregated into large clumps within hours after seeding, and increasing stirring speed did not affect the process. Efficient cell aggregation, that is, the formation of a large number of homogeneous small EBs, was achieved in the stirred bioreactor after $5 \mathrm{~d}$ of suspension culture when seeded with $1-4 \times 10^{5}$ cells $/ \mathrm{mL}$ and the stirring speed set to $25-30 \mathrm{rpm}$ (Fig. 1C). For comparison, EBs formed in the conventional static cultures were heterogeneous and showed extensive agglomeration (Fig. 1D).

Efficiency of EB Formation in Two Culture Systems The efficiency of EB formation in stirred suspension culture compared to static culture was determined by measuring the number of EBs. Stirred suspension and static culture were initiated with equal MN6 ESC numbers of $2 \times 10^{5}$ cells $/ \mathrm{mL}$. When the stirring speed was set to $25 \mathrm{rpm}$, a large number of relatively uniform EBs could be observed in stirred bioreactor after $1 \mathrm{~d}$ of suspension culture (Fig. 2A). The size of EBs formed in stirred bioreactor increased with the time of suspension culture, but EBs were relatively uniform in size and almost showed no agglomeration (Figs. 2B, C). Although a large number of EBs could also be formed in Petri dishes after $1 \mathrm{~d}$ of culture (Fig. 2D), there were extensive agglomeration of EBs leading to a number of large cell clumps during the static culture. As a result, some static EBs were irregular in shape and the differences of size of EBs were significant (Figs. 2E, F).

Stirred bioreactor could effectively prevent the agglomeration of formed EBs. The number of EBs formed in stirred bioreactor did not change significantly during the suspension 

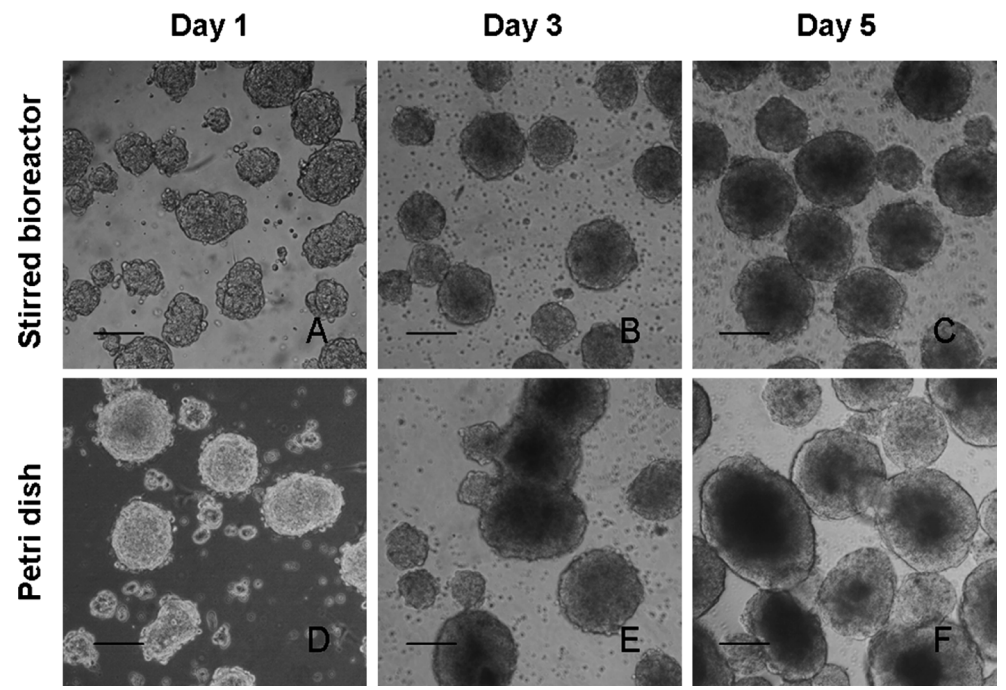

Fig. 2. Dynamic Observations of EB Formation in Stirred Bioreactors and Petri Dishes

(A-C) EBs formed in stirred bioreactor at day 1, day 3 and day 5, respectively; (D-F) EBs formed in Petri dishes at day 1, day 3 and day 5, respectively. Scale bars $=200 \mu \mathrm{m}$.
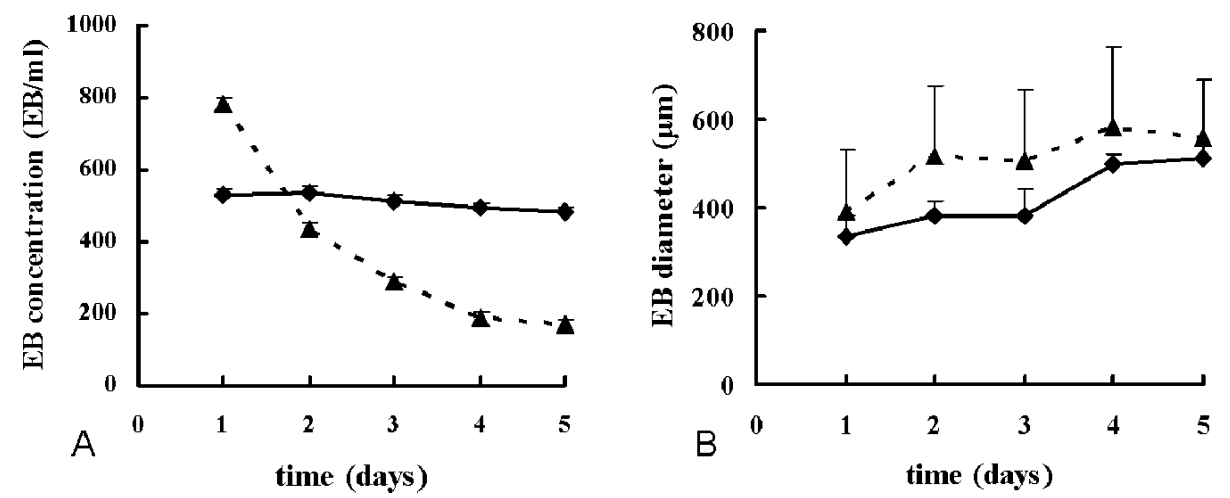

Fig. 3. Kinetics of EB Formation in Stirred Bioreactors (Solid Line) and Petri Dishes (Dash Line)

(A) Time course of EB concentration. The number of EBs formed in stirred bioreactor did not change significantly during the suspension culture, but the EB number in static culture decreased sharply after $1 \mathrm{~d}$ culture; (B) Time course of EB diameter. The size of EBs formed in stirred bioreactor increased over time and went to stable from day 4; but the size of static EBs increased rapidly due to the extensive agglomeration.
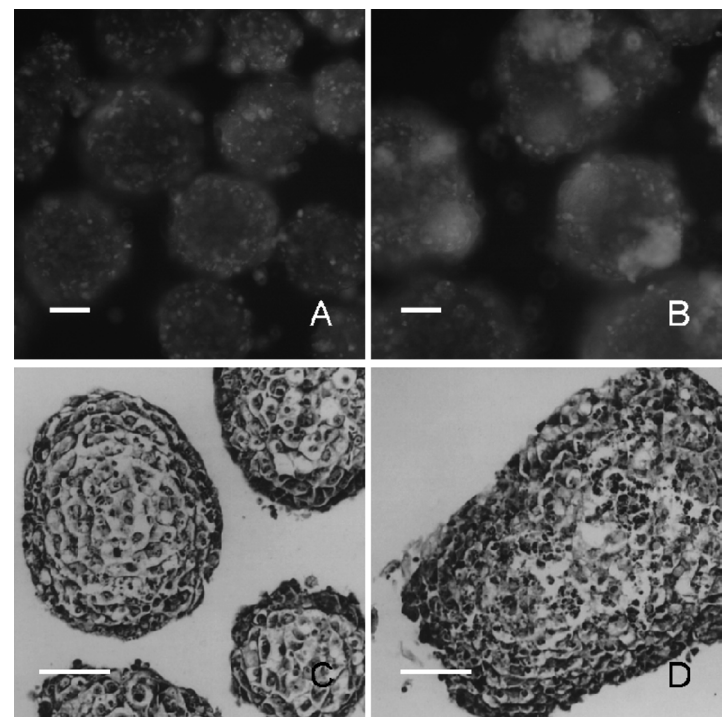

Fig. 4. Cell Viability Evaluation of EBs Formed in Stirred Bioreactor and Petri Dishes

(A,B) AO/PI staining of EBs formed in stirred bioreactor and Petri dishes, respectively; (C,D) H\&E staining of EBs formed in stirred bioreactor and Petri dishes, respectively. Scale bars $=200 \mu \mathrm{m}$. culture (Fig. 3A), but the size of EBs increased over time and went to stable from day 4 (Fig. 3B). After $5 \mathrm{~d}$ of culture, the number of EBs in stirred bioreactor was about $482 / \mathrm{mL}$, the diameter $496 \pm 20 \mu \mathrm{m}$. Although the number of EBs formed in Petri dish was far more than that of stirred bioreactor at day 1, the EB number in static culture would decreased sharply (Fig. 3A) and the size would increased rapidly (Fig. 3B) due to the extensive agglomeration of EBs. At day 5, the EB number in Petri dish was about $163 / \mathrm{mL}$, the diameter $581 \pm 168 \mu \mathrm{m}$.

Viability of EBs in Two Culture Systems Cell viability of EBs formed in the stirred suspension culture and static culture was assessed by AO/PI staining performed at day 5 . Cells within EBs from stirred bioreactor were mostly viable showing green fluorescence, while the dead cells showing red fluorescence were scarcely seen (Fig. 4A). In contrast, many cells within some EBs from static culture were stained with red fluorescence, which indicates cell death (Fig. 4B). The results of $\mathrm{AO} / \mathrm{PI}$ staining revealed that cell viability of EBs formed in stirred bioreactor appeared better than those formed in Petri dishes. Moreover, H\&E staining further confirmed that EBs formed in stirred bioreactor after $5 \mathrm{~d}$ of culture were relatively homogenous small aggregates without necrotic areas 
Oct-4

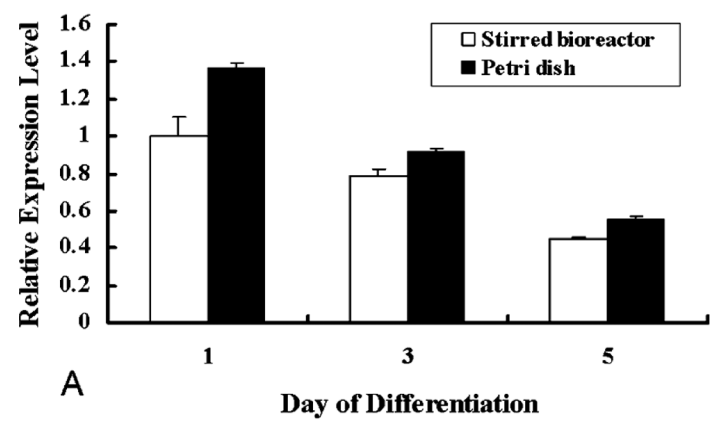

a -MHC

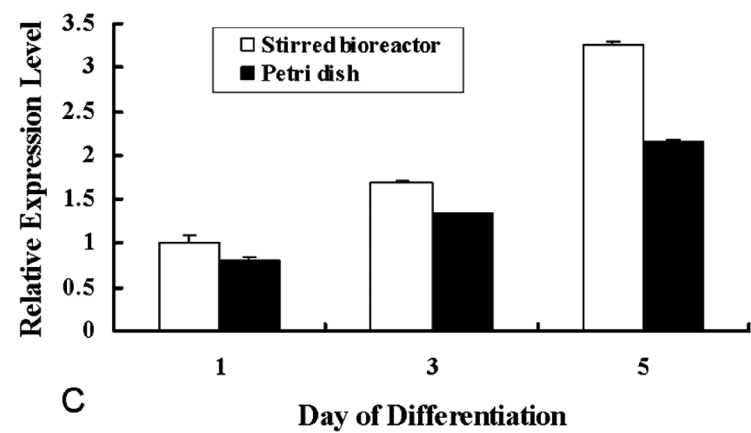

GATA-4

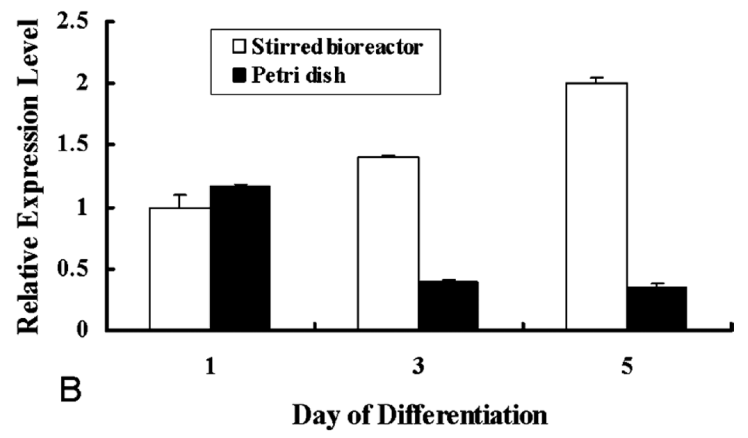

MLC-2v

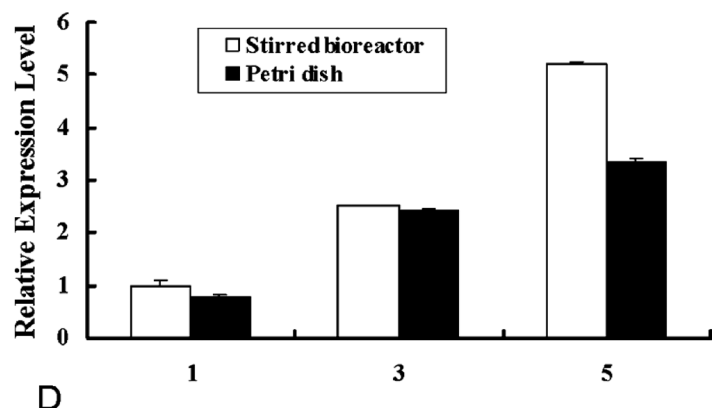

Day of Differentiation

Fig. 5. Quantitative RT-PCR Analysis of Gene Expression of EBs Formed in Stirred Bioreactor and Petri Dish

(A) Relative expression of Oct-4; (B) relative expression of GATA-4; (C) relative expression of $\alpha$-MHC; (D) relative expression of MLC-2v.
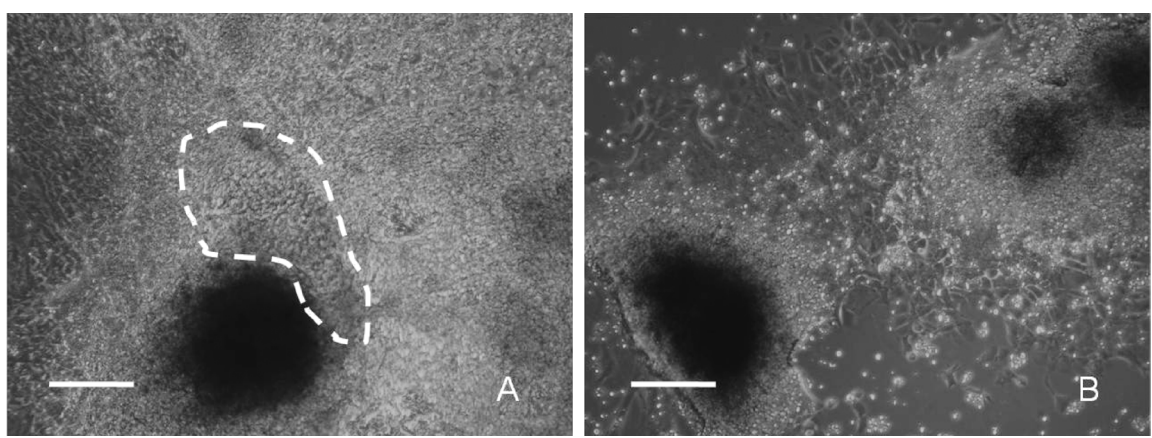

Fig. 6. The Observation of Cardiogenic Differentiation of EBs after $5 \mathrm{~d}$ of Induction with Ascorbic Acid

(A) EBs formed in stirred bioreactor contained beating cardiomyocytes foci (white dot line); (B) EBs formed in Petri dishes just grown out some differentiated cells. Scale bars $=200 \mu \mathrm{m}$

(Fig. 4C), whereas large cell clumps with necrotic cores could be found in 5-d-old EBs from Petri dishes (Fig. 4D).

Quantitative RT-PCR Analysis Gene expression of the pluripotent marker Oct-4 and cardiac differentiation was assessed quantitatively using RT-PCR. The expression of Oct-4 relative to undifferentiated ESCs were similar and no significant differences between stirred suspension and static culture, and it progressively decreased over the course of EB differentiation (Fig. 5A). Cardiac transcription factor GATA-4 of EBs in the two culture systems were expressed, but the extent of expression is very different. GATA-4 expression of EBs formed in stirred bioreactor was significantly increased over the differentiation time, while GATA-4 level of EBs formed in Petri dishes was decreased (Fig. 5B). In addition to cardiac transcription factors, EBs formed in the two culture systems also expressed transcripts for sarcomeric proteins, $\alpha$-MHC and MLC-2v. Their expression were both increased gradually with the time of differentiation, but $\alpha$-MHC and MLC-2v expressions of EBs formed in stirred bioreactor were $c a$. 1.5-fold and ca. 1.6-fold greater than that of static EBs, respectively (Figs. $5 \mathrm{C}, \mathrm{D})$. These results showed that stirred suspension culture could significantly promote the cardiac-specific gene expression of EBs.

Differentiation of EBs into Cardiomyocytes EBs formed in stirred bioreactor and Petri dish showed different adhesion capability when seeded into gelatin-coated tissue culture dishes. One day after plating onto the dishes, almost all of EBs formed in stirred bioreactor closely attached to the bottom of dishes, whereas only $80 \%$ of EBs formed in Petri dishes adhered. Under the microscope, differentiated cells could quickly grow out from EBs formed in stirred bioreactor $1 \mathrm{~d}$ after adherence; the onset of spontaneous beating was detected within stirred EBs on the second day; and most of stirred EBs contained beating cardiomyocytes at $5 \mathrm{~d}$ of induction by ascor- 
Table 2. Effect of Stirred Suspension Culture and Static Culture on Cardiogenic Differentiation of EBs

\begin{tabular}{|c|c|c|c|}
\hline Differentiation conditions & Total EBs & Number of EBs containing beating cells & Induction efficiency $(\%)$ \\
\hline Petri dishes & 415 & 64 & $15.4 \pm 1.1$ \\
\hline Stirred bioreactor & 466 & 158 & $33.9 \pm 0.6^{*}$ \\
\hline Petri dishes with ascorbic acid & 412 & 228 & $55.3 \pm 0.7$ \\
\hline Stirred bioreactor with ascorbic acid & 467 & 418 & $89.5 \pm 1.5^{*}$ \\
\hline
\end{tabular}

* Compared with Petri dishes, $p<0.05$.
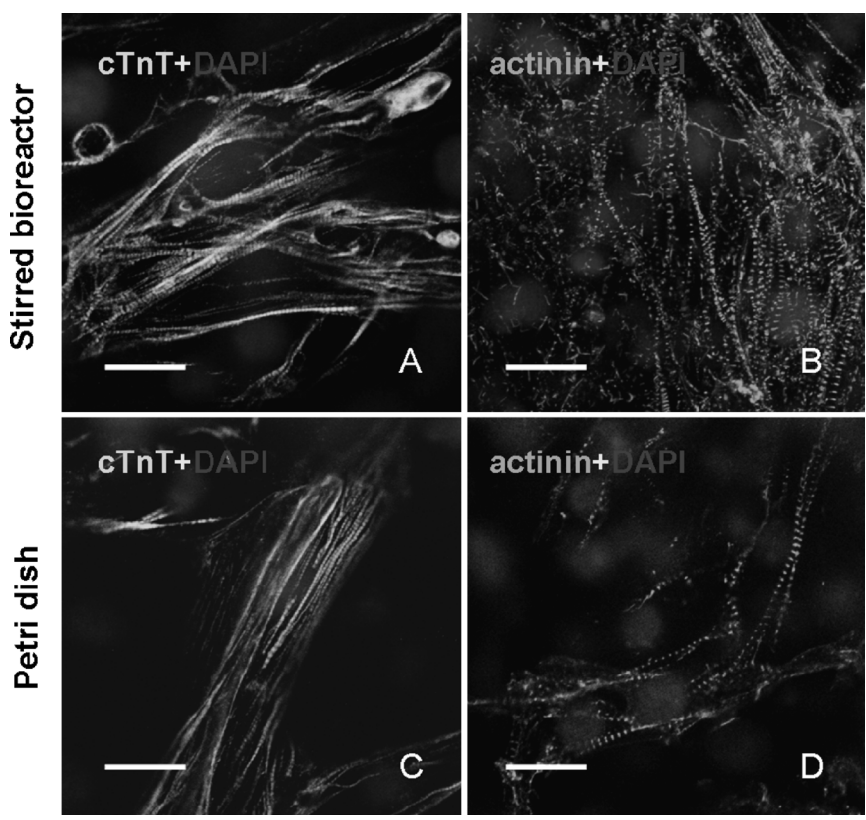

Fig. 7. Fluorescence Labeling of Cardiomyocytes within EBs Derived from Both Stirred Bioreactor and Petri Dishes

Transverse striation could be detected using cardiac troponin $\mathrm{T}$ (cTnT) (A,C) and $\alpha$-sarcomeric actinin (B,D) immunocytology. Nuclear stained by DAPI showed gray. Scale bars $=20 \mu \mathrm{m}$.

bic acid (Fig. 6A). However, cardiogenic differentiation of EBs formed in Petri dishes was delayed significantly. After $5 \mathrm{~d}$ of induction, the differentiated cells began to outgrow from static EBs (Fig. 6B).

Cardiac differentiation efficiency of EBs formed in both stirred bioreactor and Petri dish were compared and shown in Table 2. The results showed that the efficiency of cardiac differentiation of EBs formed in stirred bioreactor was up to $89.5 \%$ with the induction of ascorbic acid, and was significantly more than those formed in Petri dishes.

Immunocytochemistry and laser scanning confocal mi-
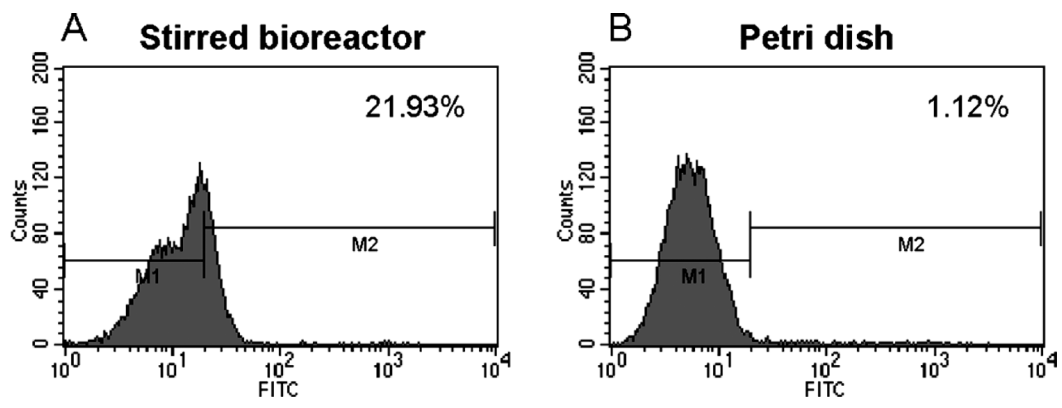

Fig. 8. Flow Cytometric Analyses of $\alpha$-Sarcomeric Actinin-Positive Cells

(A) The percent of cardiomyocytes within EBs formed in stirred bioreactor was $21.93 \%$; (B) the percent of cardiomyocytes within differentiated cells derived from static EBs was $1.12 \%$. 
Table 3. Comparison of Yields of Cardiomyocytes Generated by Stirred Suspension and Static Culture

\begin{tabular}{ccc}
\hline \hline Culture systems & Initial inoculation number of ESCs & Cardiomyocytes (CM) yield \\
\hline Stirred bioreactor $(250 \mathrm{~mL})$ & $5 \times 10^{7}$ & $5.86 \times 10^{7}$ \\
$100 \mathrm{~mm}$ Petri dish $(10 \mathrm{~mL})$ & $2 \times 10^{6}$ & $4.38 \times 10^{5}$ \\
\hline
\end{tabular}

CM/ESC: cardiomyocytes yield per input ESCs.

\section{DISCUSSION}

In this study, the effects of stirred suspension culture on the yield and viability of formed EBs, and cardiogenic differentiation of genetically modified mouse ESCs were investigated relative to static suspension culture. Stirred suspension culture could enhance the formation of EBs and increase the yield of EBs after $5 \mathrm{~d}$ of culture. EBs formed from stirred bioreactor were more uniform in size and had fewer necrotic centers, compared with the EBs from Petri dishes. Importantly, the expression of cardiac-specific genes was increased within EBs cultured in stirred bioreactor. Stirred suspension culture significantly increased the proportion of spontaneously contracting EBs with the induction of ascorbic acid and yielded a greater percentage of $\alpha$-sarcomeric actin-positive cells as detected via flow cytometry. After G418 selection, stirred suspension also yielded relatively more cardiomyocytes. The results indicated that stirred suspension culture could facilitate the growth of EBs and enhance the cardiogenic differentiation of genetically modified ESCs compared to static suspension, and thus may increase the yield of cardiomyocytes differentiated from EBs due to the increase of the overall numbers of EBs and differentiated cells.

Although spinner flasks with bulb-shaped impellers are capable of forming EBs from single-cell suspensions, spinner flasks with paddle-type impellers merely induce massive cell aggregation and EB agglomeration. ${ }^{19)}$ By optimizing cell density and stirring speed, we could successfully produce relatively uniform EBs in stirred bioreactor with paddle-type impellers. When stirred bioreactor was seeded with MN6 cell numbers of $2 \times 10^{5}$ cells $/ \mathrm{mL}$ and stirring speed was set to $25 \mathrm{rpm}$, a number of homogeneous EBs were formed after $5 \mathrm{~d}$ of culture. The yield of EBs in stirred bioreactor was about 3 times as many as EBs in Petri dish. Instead, EBs formed in Petri dishes were heterogeneous in size and showed an extensive agglomeration. This phenomenon was also reflected by the standard deviation of the diameter of EBs from the two culture systems. Mixing environment in stirred bioreactor may balance the induction of early cell aggregation to form EBs with inhibition of EB agglomeration, ${ }^{20)}$ so stirred suspension culture resulted in forming more EBs than static culture. Although previous studies have shown that stirrer vessels may have the disadvantage of generating shear forces, which would damage the cells, ${ }^{21,22)} \mathrm{AO} / \mathrm{PI}$ and $\mathrm{H} \& \mathrm{E}$ staining in our results have demonstrated that most of cells within EBs were live and there was no large necrotic centers. So we thought that shear force generated by stirred bioreactor was mild and not enough to damage cells.

A large number of EBs could be formed in static suspension, and the number of static EBs was far more than the number of EBs formed in stirred suspensinon after one day of culture, which may also be due to the stirred conditions in bioreactor. Abranches et al. showed that mouse ESCs af- ter seeding into stirred bioreactor exhibited a growth curve consisting of a death or lag phase followed by an exponential phase. $^{23)}$ The death or lag phase which occurred at the first $24 \mathrm{~h}$ might result from the adaptation of adherent ESCs to the new culture system of stirred suspension. EBs formed in static culture of Petri dishes had an extensive agglomeration from the second day, so the number of EBs was sharply declined and the size rapidly increased to form larger cell clusters. Due to nutrient or oxygen limitation, the agglomeration of static EBs ultimately resulted in massive cell death and large necrotic centers. Stirred bioreactor effectively prevented agglomeration between EBs, and the number of EBs did not change significantly during the stirred suspension process. Although the size of stirred EBs would increase over the time of suspension culture, there was no obvious necrosis because of enhanced mass transfer or gas exchange.

Because the ultimate goal was to generate ESC-derived cardiomyocytes, EBs were inducted by ascorbic acid to differentiate into cardiomyocytes after $5 \mathrm{~d}$ of suspension culture according to most of the reports that ESCs were more favorably differentiated into myocardial cells after adherent culture following suspension culture for 5 to $7 \mathrm{~d}$ to form EBs. ${ }^{24-26)}$ The quality of EBs formed in stirred bioreactor was better than that of EBs formed in Petri dish, but the differences of cardiogenic potential of EBs formed in the two culture systems are the focus of this study. Consistent with previous studies, ${ }^{13,16,17)}$ our results indicated that stirred suspension culture may create a pro-cardiogenic microenvironment within EBs that could potentially be further enhanced by additional supplementation of cardiac-inductive molecules, such as ascorbic acid. ${ }^{27)}$ In addition, ascorbic acid acts at least in part by alleviating cell senescence and accelerates gene expression changes to enhance the generation of mouse and human induced pluripotent stem cells (iPSCs). ${ }^{28)}$ Many factors of the microenvironment can simultaneously impact stem cell differentiation, including mechanical forces. ${ }^{29)}$ Nieden et al. presumed that the shear stress generated in stirred bioreactor activated Ras signaling pathway which may be contributing to the differences in proliferation and differentiation between static and stirred suspension ESC cultures. ${ }^{30)}$ Shimada et al. found that exposure to low levels of shear and microgravity within a suspension bioreactor influenced gene expression during the early development of zebrafish. ${ }^{31)}$ They concluded that cells in the developing heart were especially sensitive to this type of culture environment and attributed this characteristic to their susceptibility to morphological changes resulting from the shear forces associated with blood flow. Recent work has also demonstrated that differentiating, adherent ESCs exposed to laminar shear stress via a cone-plate viscometer exhibited significant histone modification and subsequent increases in Mef2c and $\alpha$-sarcomeric actin expression. ${ }^{32)}$ Although the specific mechanism remains unclear, the shear forces generated by stirred bioreactor provide a microenvironment that could promote the differentia- 
tion of ESCs into cardiomyocytes.

The cardiomyocytes derived from ESCs only accounted $1-5 \%$ of total differentiated cells, and the heterogenous population still contained a large number of non-myocardial cells and some of the undifferentiated ESCs which could result in the risk of tumor formation. For future cell therapy, it is very important to select and produce a very pure cardiomyocytes from ESCs. Genetic selection combined with suspension culture of stirred bioreactor could not only increase the purity of cardiomyocytes, but also increase the yield of cardiomyocytes. With optimization for parameters such as cell density, stirring speed, medium supplementation, and so on, this combination of technologies was able to produce as many as $5.86 \times 10^{7}$ cardiomyocytes in $250 \mathrm{~mL}$ stirred bioreactor. We report here a $\mathrm{CM} / \mathrm{ESC}$ of 1.17 , higher than that of static culture $(0.22$ $\mathrm{CM} / \mathrm{ESC}$ ) and that of other previous articles. ${ }^{33,34)}$ However, our results are siginificantly lower than that reported by Niebruegge et al. ${ }^{14)}$ due to variability between cell lines and culture conditions. The developmental degree of ESC-derived cardiomyocytes could not compare with adult cardiomyocytes, but ESC-derived cardiomyocytes still have some potential of proliferation. ${ }^{7)}$ Therefore, ESC-derived cardiomyocytes may have more superiority in repair capacity than other cells.

In short, the quality and cardiogenic differentiation of EBs formed in stirred suspension culture are superior to that of EBs in static culture. Stirred bioreactor was able to simply and easily achieve large-scale production of EBs, and to effectively control the agglomeration between EBs. We have established a method for effective generation of pure ESC-derived cardiomyocytes, which not only provide a cell source for tissue engineering and regenerative medicine, but also could be used to investigate cell cycle regulation, development research, drug screening, and cardiac electrophysiology study in vitro. Future studies in human ESCs or iPSCs will be necessary to advance this technology for potential regenerative therapy.

Acknowledgements This work was supported by the National Basic Science and Development Programme (2012CB518103 and 2012CB518105), the National Natural Science Foundation of China (No. 81121004 and 31100705), and the Eleventh Five-year Program for Young Scholar Project in Military, China (No. 06Q86). We are grateful to Shujun Liu and Maixing Ai for excellent technical assistance.

\section{REFERENCES}

1) Evans MJ, Kaufman MH. Establishment in culture of pluripotential cells from mouse embryos. Nature, 292, 154-156 (1981).

2) Kehat I, Khimovich L, Caspi O, Gepstein A, Shofti R, Arbel G, Huber I, Satin J, Itskovitz-Eldor J, Gepstein L. Electromechanical integration of cardiomyocytes derived from human embryonic stem cells. Nat. Biotechnol., 22, 1282-1289 (2004).

3) Rubart M, Pasumarthi KB, Nakajima H, Soonpaa MH, Nakajima HO, Field LJ. Physiological coupling of donor and host cardiomyocytes after cellular transplantation. Circ. Res., 92, 1217-1224 (2003).

4) Pal R. Embryonic stem (ES) cell-derived cardiomyocytes: a good candidate for cell therapy applications. Cell Biol. Int., 33, 325-336 (2009).

5) Pucéat M. Protocols for cardiac differentiation of embryonic stem cells. Methods, 45, 168-171 (2008).

6) Sarkar P, Rao BM. Molecular aspects of cardiac differentiation in embryonic stem cells. Crit. Rev. Biomed. Eng., 37, 283-320 (2009).

7) Xu C, Police S, Rao N, Carpenter MK. Characterization and enrichment of cardiomyocytes derived from human embryonic stem cells. Circ. Res., 91, 501-508 (2002).

8) Segev H, Kenyagin-Karsenti D, Fishman B, Gerecht-Nir S, Ziskind A, Amit M, Coleman R, Itskovitz-Eldor J. Molecular analysis of cardiomyocytes derived from human embryonic stem cells. Dev. Growth Differ., 47, 295-306 (2005).

9) Klug MG, Soonpaa MH, Koh GY, Field LJ. Genetically selected cardiomyocytes from differentiating embronic stem cells form stable intracardiac grafts. J. Clin. Invest., 98, 216-224 (1996).

10) Dang SM, Kyba M, Perlingeiro R, Daley GQ, Zandstra PW. Efficiency of embryoid body formation and hematopoietic development from embryonic stem cells in different culture systems. Biotechnol. Bioeng., 78, 442-453 (2002).

11) Gerecht-Nir S, Cohen S, Itskovitz-Eldor J. Bioreactor cultivation enhances the efficiency of human embryoid body ( $\mathrm{hEB}$ ) formation and differentiation. Biotechnol. Bioeng., 86, 493-502 (2004).

12) Cameron CM, Hu WS, Kaufman DS. Improved development of human embryonic stem cell-derived embryoid bodies by stirred vessel cultivation. Biotechnol. Bioeng., 94, 938-948 (2006).

13) Lü S, Liu S, He W, Duan C, Li Y, Liu Z, Zhang Y, Hao T, Wang Y, Li D, Wang C, Gao S. Bioreactor cultivation enhances NTEB formation and differentiation of NTES cells into cardiomyocytes. Cloning Stem Cells, 10, 363-370 (2008).

14) Niebruegge S, Nehring A, Bär H, Schroeder M, Zweigerdt R, Lehmann J. Cardiomyocyte production in mass suspension culture: embryonic stem cells as a source for great amounts of functional cardiomyocytes. Tissue Eng. Part A, 14, 1591-1601 (2008).

15) Fok EY, Zandstra PW. Shear-controlled single-step mouse embryonic stem cell expansion and embryoid body-based differentiation. Stem Cells, 23, 1333-1342 (2005).

16) Carpenedo RL, Sargent CY, McDevitt TC. Rotary suspension culture enhances the efficiency, yield, and homogeneity of embryoid body differentiation. Stem Cells, 25, 2224-2234 (2007).

17) Sargent CY, Berguig GY, McDevitt TC. Cardiomyogenic differentiation of embryoid bodies is promoted by rotary orbital suspension culture. Tissue Eng. Part A, 15, 331-342 (2009).

18) He WJ, Li SC, Ye LL, Liu H, Wang QW, Han WD, Fu XB, Chen ZL. Genetic enrichment of cardiomyocytes derived from mouse embryonic stem cells. Afr. J. Biotechnol., 10, 5683-5691 (2011).

19) Schroeder M, Niebruegge S, Werner A, Willbold E, Burg M, Ruediger M, Field LJ, Lehmann J, Zweigerdt R. Differentiation and lineage selection of mouse embryonic stem cells in a stirred bench scale bioreactor with automated process control. Biotechnol. Bioeng., 92, 920-933 (2005).

20) Dang SM, Gerecht-Nir S, Chen J, Itskovitz-Eldor J, Zandstra PW. Controlled, scalable embryonic stem cell differentiation culture. Stem Cells, 22, 275-282 (2004).

21) Chisti Y. Hydrodynamic damage to animal cells. Crit. Rev. Biotechnol., 21, 67-110 (2001).

22) Hammond TG, Hammond JM. Optimized suspension culture: the rotating-wall vessel. Am. J. Physiol. Renal Physiol., 281, F12-F25 (2001).

23) Abranches E, Bekman E, Henrique D, Cabral JM. Expansion of mouse embryonic stem cells on microcarriers. Biotechnol. Bioeng., 96, 1211-1221 (2007).

24) Boheler KR, Czyz J, Tweedie D, Yang HT, Anisimov SV, Wobus AM. Differentiation of pluripotent embryonic stem cells into cardiomyocytes. Circ. Res., 91, 189-201 (2002).

25) Sachinidis A, Fleischmann BK, Kolossov E, Wartenberg M, Sauer $\mathrm{H}$, Hescheler J. Cardiac specific differentiation of mouse embryonic stem cells. Cardiovasc. Res., 58, 278-291 (2003).

26) Heng BC, Haider HK, Sim EK, Cao T, Ng SC. Strategies for directing the differentiation of stem cells into the cardiomyogenic lineage in vitro. Cardiovasc. Res., 62, 34-42 (2004).

27) Takahashi T, Lord B, Schulze PC, Fryer RM, Sarang SS, Gullans 
SR, Lee RT. Ascorbic acid enhances differentiation of embryonic stem cells into cardiac myocytes. Circulation, 107, 1912-1916 (2003).

28) Esteban MA, Wang T, Qin B, Yang J, Qin D, Cai J, Li W, Weng Z, Chen J, Ni S, Chen K, Li Y, Liu X, Xu J, Zhang S, Li F, He W, Labuda K, Song Y, Peterbauer A, Wolbank S, Redl H, Zhong M, Cai D, Zeng L, Pei D. Vitamin C enhances the generation of mouse and human induced pluripotent stem cells. Cell Stem Cell, 6, 71-79 (2010).

29) Metallo CM, Mohr JC, Detzel CJ, de Pablo JJ, Van Wie BJ, Palecek SP. Engineering the stem cell microenvironment. Biotechnol. Prog., 23, 18-23 (2007).

30) zur Nieden NI, Cormier JT, Rancourt DE, Kallos MS. Embryonic stem cells remain highly pluripotent following long term expansion as aggregates in suspension bioreactors. J. Biotechnol., 129,
$421-432$ (2007).

31) Shimada N, Sokunbi G, Moorman SJ. Changes in gravitational force affect gene expression in developing organ systems at different developmental times. BMC Dev. Biol., 5, 10 (2005).

32) Illi B, Scopece A, Nanni S, Farsetti A, Morgante L, Biglioli P, Capogrossi MC, Gaetano C. Epigenetic histone modification and cardiovascular lineage programming in mouse embryonic stem cells exposed to laminar shear stress. Circ. Res., 96, 501-508 (2005).

33) Zandstra PW, Bauwens C, Yin T, Liu Q, Schiller H, Zweigerdt R, Pasumarthi KB, Field LJ. Scalable production of embryonic stem cell-derived cardiomyocytes. Tissue Eng., 9, 767-778 (2003).

34) Bauwens C, Yin T, Dang S, Peerani R, Zandstra PW. Development of a perfusion fed bioreactor for embryonic stem cell-derived cardiomyocyte generation: oxygen-mediated enhancement of cardiomyocyte output. Biotechnol. Bioeng., 90, 452-461 (2005). 